International Journal of Engineering \& Technology, $7(2.28)(2018) 160-163$
International Journal of Engineering \& Technology
SPC
Website: www.sciencepubco.com/index.php/IJET
Research paper

\title{
On approach to the agreement of diverse stakeholders' interests and goals in the governance
}

\author{
Zinaida K. Avdeeva*, Svetlana V. Kovriga \\ Trapeznikov Institute of Control Sciences of RAS, Moscow, Russia \\ *Corresponding author E-mail: zinaida.avdeeva@gmail.com
}

\begin{abstract}
The paper proposes the use of a collective cognitive map when solving governance problems as an effective means of diverse stakeholders' interests and goals agreement. For compose such a map, an original approach is proposed that combines (1) the clarification and agreement of stakeholder representations on the governance problem using a number of criteria for improving the map quality and (2) the clusterization of similar stakeholder representations.
\end{abstract}

Keywords: Governance; Stakeholders; Decision Making; Collective Cognitive Map

\section{Introduction}

\subsection{The problem statement}

Modern decision maker's situations are characterized not only by rapid variability, by the interaction of many diverse and interdisciplinary factors, but also by the presence of active stakeholders whose beliefs and interests lead to variety of the directions of the situation development.

Therefore, in the area of public administration and government policy making governance in condition of strategic development becomes increasingly important along with traditional management.

Unlike management, governance focuses on other activities, mainly related to goal-setting and monitoring. Governance is about negotiating and deciding amongst different stakeholders' value interests.

Governance ensures that stakeholder needs, conditions and options are evaluated to determine balanced, agreed-on system objectives (goals) to be achieved; setting direction through prioritization and decision making; and monitoring performance and compliance against agreed-on direction and objectives [1].

The stakeholder's community of consists of individuals and groups that have not only different interests, but also different potential of positive or negative influence on the joint activities outcome. At the same time, their interaction at the level of public administration has often a distributed nature. In these conditions, the role of governance networks increases.

According to [2], a governance network is a temporal institutionalization of a forum of interdependent but operationally autonomous and self-governing actors who collaborate in a shared effort to achieve agreed goals.

It is important to note that substantive complexity within governance networks is not so much caused by the complicatedness of problems and lack of information and knowledge, how many lack of consensus on the nature of problems, their causes and solutions [2]. Governance problem solving, policymaking involve with diverse stakeholders. They have different perceptions of the situation and also interpret available information differently.

Therefore, collecting information and tapping into expert knowledge cannot solve the complex problems in conditions when they are interpreted in different ways.

Thus, one of the most important means of improving the quality of governance activities is the integration of information and communication technologies with the latest achievements in the area of expert analytical methods and technologies of goal setting, monitoring and analysis of situations that take into account the listed specifics of stakeholder interaction in solving governance problems.

\subsection{The proposed approach to the problem solution}

In the modern decision making methodology, methods of cognitive mapping are being developed.

The application area of cognitive maps covers complex and illstructured systems and situations, including situations related to stakeholders.

A cognitive map of the situation is understood as a formalized model of the situation, reflecting the knowledge and or beliefs of the subject, individual or collective, about the causal (or, causeeffect) influences between the significant factors of the situation.

The application spectrum of cognitive maps spreads from conceptual modelling, aimed at improving the structuring and understanding of problems (see, e.g., [4]), before to solving practical problems of analysis and modelling the situation dynamics (see, e.g., [5]).

In the first case, a total and agreement representation of the problem situation is constructed in the form of a weakly formalized cognitive map.

In the latter case, so-called formal cognitive maps (FCMs) are used. The Figure 1 shows a fragment of such map (a map of the 
enterprise development with environmentally dangerous production).

Formally, the obligatory base of the FCMs family is a directed graph, which nodes are associated with factors (or concepts) and arches are interpreted as direct causal influences (or causal relations, connections, links) between factors. Usually arches are attributed with influence signs ("+" or "-") or influence weights (intensities).

In addition to listed constructs, i.e. factors, which are represented as variables, and their links, the prevailing role in definition of behavior is played by one more construct, which we refer to as a bundle, i.e. a factor with all incoming direct influences with the associated function, sometimes referred to as the influence aggregation function. (Weights are parameters of a specific type of the function.) So the FCMs allow for formal processing [6]. In the paper, we focus on FCMs.



Link: derect causal influence

(+): increase (decrease) in the factor 8 causes increase (decrease) in the factor 2 (): increase (decrease) in the factor 7 casuses decrease (increase) in the foctor 2 (other things being equel)

Fig.1: Fragment of a cognitive map of the enterprise development with environmentally dangerous production

In our study, we propose the CCM use to improve governance effectiveness. In this context, the CCM is seen as a means (1) of agreeing the stakeholders' opinions, (2) of their total representation of the governance problem, (3) of identifying possible conflicts of interest and seeking their solution. In this case, the CCM can reflect not only the opinions of stakeholders, but and the knowledge of multidisciplinary team of experts to complete representation of the situation.

In the stakeholder theory [7], in analyzing the business situation, different categories of stakeholders are distinguished: internal and external, primary and secondary. The selection basis is the two main attributes: the degree of the relationship of stakeholder interests with the strategic goals of the organization's development and the degree of their influence on the achievement of these goals The internal primary stakeholders are those whose interests are directly related to the organization goals (e.g., owners, managers, employees). The stakeholders of the external environment in relation to the organization can be primary (e.g., consumers, competitors) or secondary (e.g., government).

In our study, we focus on agreeing the interests of the community of primary stakeholders, connected with the identified governance problem and interested in its solution. The secondary stakeholders' influence (in terms of [7]) is also taken into account in the CCM based on the additional knowledge of multidisciplinary team of experts.

To compose such a map, we propose original approach, combining (1) the clarification and agreement of stakeholder representations on the governance problem using a number of criteria for improving the CCM quality and (2) the clusterization of similar stakeholder representations.

\section{Collective cognitive map construction}

\subsection{The state of the art. The brief description}

An analysis of recent publications ([5], [7]-[10]) on approaches to composing a CCM shows the following.

- CCMs can be divided into two categories on the basis "whose knowledge is being structured?" [9]. The first category includes traditional CCMs. Given the lack of objective data about the problem situation, such a CCM is represented as a "complete" model reflecting the knowledge of a multidisciplinary team of traditional experts. Here, the "complete" model is understood as a model sufficient to represent the problem situation from the point of view of the experts. Accordingly, the more the diverse experts are involved, the more different aspects of the situation are reflected in the CCM.

According to [9] the second category of CCM appeared recently. It is connected with the need to include in the model of representations of so-called non-traditional experts - stakeholders. These CCMs are often used in the context of planning and management, decision-making in different areas (e.g., environment [9], corporate business [10]).

The distinctive feature of the second category is that the CCM reflects the stakeholders' representations of the problem, taking into account their interests, goals, vision of possible ways of the situation development. It is important here to get an agreed representation on the problem and its solution.

Further for brevity in the paper, if the difference between experts and stakeholders is not significant, the term "actors" is used.

- In the cognitive mapping, two approaches to composing CCM are common [5], [9]: the aggregation technique, when individual maps are formed, and then aggregated into a CCM and collective technique, when a general map is constructed in a group discussion (using methods brainstorming, focus groups, etc.).

The choice of technique is related to the intended use of the CCM. The aggregation provides a comprehensive problem situation representation, taking into account the diversity of individual representations of it. The collective approach is aimed, first of all, at achieving a common understanding in the team on the problem situation.

In the area of FCM application the aggregation technique prevails. Here the typical function of the formal "agreement of points of view" in the CCM is the integration of several cognitive maps by averaging the influence weights between the same factors [5].

Human processes of agreeing knowledge and representations of actors, as far as can be judged from publications, are not supported today by any systematic methods. In practice, it is carried out by moderators, based on their experience [9]. Here and below, the moderator is an actor who owns the cognitive mapping methodology.

- Finally, it is important to highlight the problem of risks for the validity of results due to the human factor in FCM application. It consists in the inevitable and significant participation of people (experts, stakeholders, moderators) in solving problems through formal methods. The practical significance of this problem in cognitive mapping is justified and empirically confirmed[6], [11], [12]. Not only do researchers in cognitive mapping directly or indirectly talk about risks for validity (or directly errors), but also in the related area of system dynamics [13], [14]; different approaches are proposed to reduce the role of risks in the construction of cognitive maps.

However, this problem is practically not considered in the CCMs context. Among the few publications, it is possible to single out the researches of T. Bouzdine-Chameeva (e.g., [15]), which raises the question of the dependence of the CCM validity on the concepts' system used in the formalization of expert representations. A. McLucas, a system dynamics specialist [14], singles out the agreement of points of view as a significant means of supporting validity, in particular, with regard to the evaluation of the factor 
values when initiating an imitative model of causal influences. But the listed authors in their papers do not give any practical recommendations on the indicated problem.

\subsection{The main ideas of our approach}

Our approach to composing of formal CCMs is as follows.

- Rejection of the typical procedure for the CCM construction: a formal integration of several cognitive maps by averaging the influence weights. It is caused by a lack of knowledge of acceptable validity necessary for estimating influences' weights in the map (confirmed by studies on the expert estimation problem in cognitive mapping [12]).

- The CCM formation is proposed to begin with the construction of sign cognitive maps before estimating the influences' weights in them. Our practical experience shows that in order to increase the validity of the estimation of the influences' weights, the quality parameters spectrum of the sign map, such as the comprehensibility of factors, links, etc., plays a decisive role.

In sign cognitive map, only the signs of causal influences are given: $(+)-$ a positive influence or $(-)-$ a negative influence. (The fragment of the map (Fig. 1) can be represented in the form of a sign map, if instead of the influence weights leave the corresponding signs of influence.)

Each such sign cognitive map $K^{l}$ reflects the representation of a stakeholder 1 (or group, in the case of a joint construction) on the governance problem.

Among actors, there must be a moderator who manages this process with the purpose of

- achievement of an acceptable intuitive understanding and consistent representation of the problem situation by the stakeholders; here by "acceptability" is understood such a level at which a collective solution of the problem situation is possible without taking into account the existing differences in the understanding of the situation by different stakeholders;

- decreasing of a negative distorting effect when translating primary representations into a formalized situation model in the language of a formal model (selected type of FCM).

In the latter case, it is a question of two interpretations of the same formal map: intuitive and mathematical ones (the latter being formally processed later to produce new knowledge) with more or less distortion between them. In particular, these or other unacceptable or risky distortions arise when translating the primary knowledge about the problem situation into the elementary constructs of a map [16]. The Fig. 1 shows the elementary constructs of a map.

Our hypothesis is that when compositing maps on the basis of structuring and agreeing stakeholder representations, one can rely on a criteria family of the absence of risks for the validity of the end results that has been developing in our studies, starting from [11] and up to the present day.

The criteria family forms an open system that reflects the logic of composing cognitive maps from elementary language constructs (factors-variables, direct causal links and bundles (Fig. 1)), up to the criteria applicable to more complex constructs.

With regard to capabilities of estimation of conformity, the proposed criteria are not formal. This means that decision on conformity is made by the actor composing a map independently or with the help of the moderator carrying out control of formalization correctness. In order to facilitate understanding, criteria are provided in the form of statements in natural language.

The efficiency of these criteria is empirically confirmed by the improvement of the cognitive maps' quality that were built in the process of cognitive mapping teaching (i) students who studied systems modelling technologies; (ii) experts and analysts in the area of public administration.

This study showed that the application of this approach requires the development of typical techniques of teaching cognitive mapping (with the support of the appropriate software) for the various categories of actors involved in the decision-making process in governance.

- Next, the formed stakeholder maps $\left\{K^{l}\right\}$ are integrated into CCM (possibly in several CCMs) based on the principle of their representations proximity on the governance problem. To do this, we propose a formal procedure for clustering maps $\left\{K^{l}\right\}$ based on the proximity of the matrices associated with these maps (proximity measures are selected according to known functions used in multiparameter classification [17]).

Accordingly, one or more clusters $\left\{C_{k}\right\}$ are formed, each of which includes stakeholder cards with similar representations on the governance problem. In each $C_{k}$, a median map $K^{C_{k}}$ of the cluster is formed by integrating the maps $\left\{K^{l}\right\}^{C_{k}}$ included into it based on the criterion of the stakeholder interest proximity. The median maps agreement procedure includes the determination of significant differences between them, integrating them into the total situation map in the case of weak differences and the conflict analysis in the case of significant differences [18].

In general, several CCMs can be obtained. The formation of several CCM allows you to take into account alternative views about the governance problem. Thus, the application of this approach avoids the "averaging" (i.e., ignoring the differences) of the actors' opinions used in modern aggregating procedures of cognitive maps.

Further on each such CCM (after estimating the influence weights), control problems can be posed and solved using the techniques of statement and solving of situation development control problems under the influence of external environment using the SWOT-analysis and situation control problems with many active stakeholders [19].

\section{Conclusion}

The proposed approach to agreement the stakeholders' interests is focused on teamwork on complex issues or formation and argumentation of the strategy goals of development in the area of public administration and government policy making with the involvement of various sectors of civil society.

Currently, our efforts are aimed at implantation this approach in the author technology of the strategic goal-setting and monitoring of a complex system development [19]. The Figure 2 shows the general scheme of strategic goal-setting and monitoring of a system development, implemented by technology.

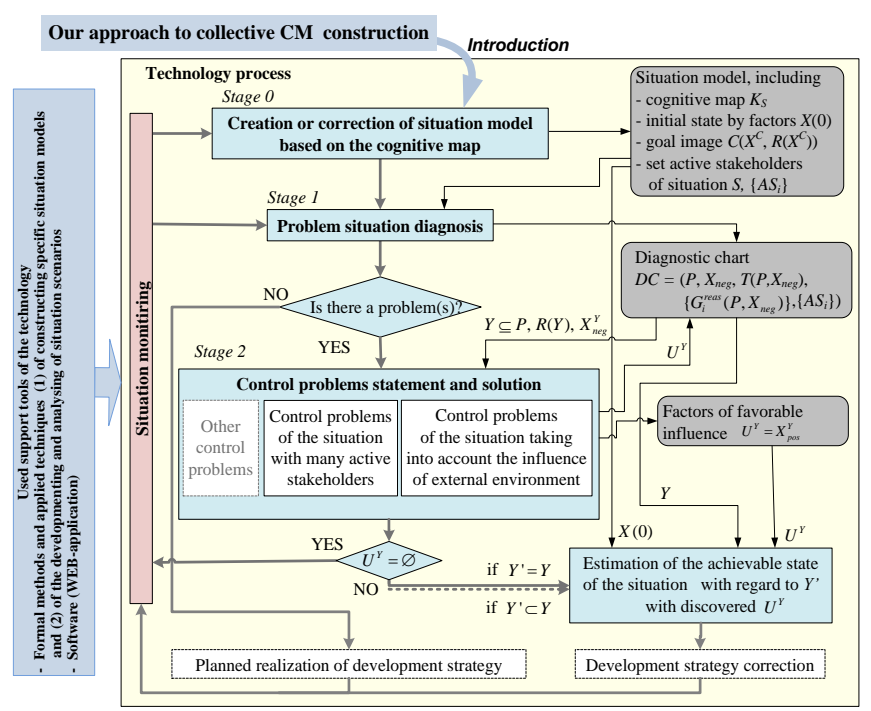

Fig. 2: The general scheme of strategic goal-setting and monitoring of a system development 
Today, the technology includes an open set of approaches and techniques aimed at early stages of solving system development problems in ill-structured situations: diagnosing problems and dynamic goal-setting in the framework of strategic planning and management.

Dynamic goal-setting supposes the formation and argumentation of the strategic goals of the system development and the directions of their achievement; and the possibility of their correction based on the monitoring of the situation.

The technology is applicable to objects of different scale (enterprise, corporation, region, state, military organization, etc.).

Used support tools of the technology include

- formal methods and applied techniques (1) of constructing specific situation models and (2) of the development and analysing of situation scenarios;

- software (WEB-application).

\section{Acknowledgement}

This research was partial supported by the Russian Science Foundation (grant № 17-18-01326).

\section{References}

[1] COBIT 5: A Business Framework for the Governance and Management of Enterprise IT. ISACA, 2012.

[2] Sørensen E. (2014), The metagovernance of public innovation in governance networks. Proceedings of the Policy \& Politics conference: The challenges of leadership and collaboration in the 21st Cen tury, available online: https://www.bristol.ac.uk/media-library/ sites/sps/migrated/documents/sorensonthemetagovernanceofpublicinn ovation.pdf, , last visit: 19.04.2018.

[3] Klijn E., Koppenjan J. (2014), Complexity in governance network theory. Complexity, Governance \& Networks, Vol. 1, No.1, pp. 6170, available online: https://ubp.uni-bamberg.de/ojs/index.php/ cgn/article/view/20, last visit: 19.04.2018.

[4] Howick S. and other (2008), Building confidence in models for multiple audiences: the modelling cascade. European Journal of Operational Research, Vol.183, No. 3, pp. 1068-1083.

[5] Fuzzy cognitive maps advances in theory, methodologies, tools and applications. M. Glukas (Ed) (2010). Springer-Verlag Berlin Heidelberg.

[6] Abramova N. and other (2010), Subject-formal methods based on cognitive maps and the problem of risk due to the human factor. Cognitive maps. IN-TECH, pp. 35-62.

[7] Freeman R. and other (2010), Stakeholder theory. The state of the art. Cambridge University.

[8] Kjærgaard A., Blegind J. (2012), Using cognitive mapping to represent and share users' interpretations in technology adaptation. Proceedings of the New frontiers in management and organizational cognition conference. National University of Ireland Maynooth.

[9] Gray S.A., Zanre E. and Gray S. (2014), Fuzzy cognitive maps as representations of mental models and group beliefs: theoretical and technical issues. Fuzzy cognitive maps for applied sciences and engineering. E. Papageorgiou (Ed). Springer, pp. 29-48.

[10] Garoui N., Jarboui A. (2012), Cognitive governance, cognitive mapping and cognitive conflicts: structural analysis with the MICMAC method, Cogent Economics \& Finance, No. 2, available online: https://www.tandfonline.com/doi/full/10.1080/23322039. 2014.922893, last visit: 19.04.2018.

[11] Abramova N., Kovriga S. (2011), The expert approach to verification at cognitive mapping of ill-structured situations. Proceedings of 18th IFAC World Congress, pp. 1997-2002.

[12] Abramova N., Telitsyna T. (2013), An approach to analysis of expert estimation validity in cognitive mapping. Proceedings of the IFAC Conference on manufacturing modelling, management, and control (MIM2013), pp. 927-932.

[13] Burns J., Musa P. (2001) Structural validation of causal loop diagrams. Proceedings of the System dynamics society 19th annua conference, available online: https://www.researchgate.net/publi caton/241870937_Structural_Validation_of_Causal_Loop_Diagram s, last visit: 19.04.2018.

[14] McLucas A. (2002) Improving causal mapping practice using the system dynamics "front-end" tool. Proceedings of the 20th International System Dynamics Conference.
[15] Bouzdine-Chameeva T. (2007), The ANCOM-2 solution to support knowledge work. International Business Management, Vol.1 No. 2 pp. 12-19.

[16] Abramova N. (2012), Interdisciplinary approach to verification in decision-making with formal methods. Handbook on psychology of decision-making: new research, K. Moore, N. Gonzalez (eds), Nova Science Pub Inc, pp. 89-111.

[17] Mirkin B. (1996) Mathematical classification and clustering. Springer US.

[18] Avdeeva Z., Kovriga S. (2014), On some principles and approaches to the construction of collective cognitive maps of situations Large-Scale Systems Control, Vol. 52, pp. 37-68 (published in Russia).

[19] Avdeeva Z., Kovriga S. (2017), The technology of the strategic goal-setting and monitoring of a system development on the basis of cognitive mapping. Procedia Computer Science, Vol. 122, pp. 977-984 